\title{
An Abstract Model for Incentive-Enhanced Trust in P2P Networks
}

\author{
Mats Neovius \\ Department of Computer Science, Åbo Akademi University, \\ Lemminkäisenkatu 14, FIN-20520 Turku, Finland \\ mats@neovius.com
}

\begin{abstract}
Peer-to-Peer (P2P) networks have emerged as a prime research topic, partly due to the vast unexploited possibilities unrestricted distribution of the workload provides. The main hindrance for unrestricted exploitation of the P2P topology is, due to lack of security-related issues, the gullible attitude taken towards unknown agents. Therefore, the severity of the vulnerabilities caused by gullibility must be mended by other means, for example, by an effective incentive scheme encouraging agents to trustworthy behaviour. This paper presents an abstract model for incentive enhanced trust, to progressively assign the participating agents rights for accessing distributed resources, emphasising consistent behaviour. The model consists of a degrading formula, an illustrative incentive triangle and a best-effort distributed supervision model. Moreover, the same incentive model facilitates anticipation of future behaviour concerning any given agent founded on several distinct agents' opinion, suggesting that any knowledge concerning the counterpart is better than none.
\end{abstract}

Keywords: Peer-to-Peer networks, incentive, trustworthiness, anticipation.

\section{Introduction}

Reputation-based trust systems are widely studied and are probably the most realistic approach to anticipate future behaviour of an agent. Consequently, as in reality, there must exist a powerful incentive encouraging participants in a P2P network to credibly exchange information and act consistently benevolently. Thus, as mentioned by Kamvar, Schlosser and Garcia-Molina in [1], the identification must be a long-term user-specific, not relying on an externally assigned identity such as the IP address.

One way to encourage consistent behaviour is by assigning a covetous benefit to agents behaving benevolently. This gain should play the role of real-life money; it should be desirable and entitle to additional privileges. However, such an advantage attracts fraud in various forms. To describe the problems, it is essential to declare the basic frameworks and concepts which trust, in this case, is to be applied on.

\subsection{Peer-to-Peer Networks}

A P2P system implementing trust resembles inter-human communication in many ways. In a P2P network, all participating agents act as clients as well as servers and 
possess equal rights, which suggest to a self-policing structure. Therefore, the definition concerning $\mathrm{P} 2 \mathrm{P}$ architecture to be used throughout this paper is as follows:

A P2P architecture is a distributed network where each node grants other requesting nodes access to its shared resource(s). The resource(s) is/are accessible by any other participant on the network directly without intermediate servers.

Consequently, this paper views the participants in a P2P network as "members of a society", where an agent's actions are egocentrically determined by benefit. Moreover, a P2P system should be capable of handling any arbitrary agent's unexpected drop-off from the network at any given time, without the network suffering any loss of service [2]. This excludes implementation of a predefined structure, such as servers or pre-shared secrets.

Despite the exclusion of central units, we argue that deploying an incentive in an agent-centric P2P architecture, a structured overlay network is a necessity. This is motivated because it enables systematic knowledge lookup, efficient collaboration between the participating agents for maintaining the incentive and assignment of credit to the appropriate agent. This paper considers the overlay system organised as a Pastry Distributed Hash Table (DHT) architecture. The Pastry DHT system provides scalability, low network diameter and proximity selection $[3,4]$.

\subsection{The Trust Metric}

Trust is a social phenomenon and can only exist between two distinct matters of which at least one is capable of feeling. As such, all models of trust should be based on the same as the social trust, knowledge about the counterpart. This paper discusses unrestricted agent-centric trust and situations where it is assumed that the counterpart is behaving irrationally. Walsh and Sirer in [5] propose an object centric reputation scheme that is restricted to a specific kind of objects, in their case files. Such a system is however, unsuitable for agent-centric reputation evaluation because peers' behaviour vary.

Implementing trust to be processed in a microprocessor requires that it can be measured and thereby, compel assigning a value for the metric. In a binary formation, an agent is evaluated as either trustworthy (affirmative) or untrustworthy (negative). Eventually every assessment should fit the binary formation. Considering the perpetually changing environment and variety of levels demanded, binary formation is insufficient for comprehensive usage. Therefore, the trust metric is considered in this paper discrete, between 0 (none) and 1 (complete), with a sufficient amount of states. According to calculations made on the values, the trustor will assign the trustee-specific rights to access and/or exploit resource(s), as will the trustee select the provider.

Besides the value of the metric, it must be distinguishable on a per actor basis and thus, explicitly mapped to a unique ID, as humans are recognised by characteristics such as the voice, by sight etc. Consequently, we argue that a unique ID is a precondition for implementing trust of any kind between conditionally trustworthy matters. 


\subsection{Recognised Abuses in P2P Networks}

The present gullible approach adapted by participants in a P2P network, and the limited possibilities to locate colluding agents, attracts abuses of many kinds. Concerning peer misbehaviours, three types are recognised: collude inflation, deflating and faking [6].

Collude inflation are situations where a conglomerate of agents collaborate by reporting positively about each other in order to achieve a higher trustworthiness value. The problem is present in centralised online auctions and in reality, because there is no way to verify the feedback's truthfulness and dignity. However, including only one report per agent such as in eBay.com [7] and degrading the information by time would hamper any colluding intentions.

Deflation is a situation where a set of agents defames another's reputation by reporting unsatisfactory behaviour concerning it. This is comparable to spreading rumours in reality. However, degrading of reports as for collusion, affects deflation equally and is a feasible countermeasure.

A faker is an agent that introduces itself as another agent (usually) possessing a higher reputation. This problem should be solved at the assignment of the ID or at the mapping of the feedbacks to an ID. However, this is out of the scope of the topic and this paper assumes that the ID's are unique and the feedbacks are authentic.

Besides the misbehaviours concerning reputations; annoyances such as "freeriding" and "tragedy of the commons" are widely acknowledged. Both are consequences of unfair exploitation and contribution respectively, of the commonly accessible resources and can be solved utilising the kind of incentive presented later in this paper.

\section{Trust in an Open Environment}

A trust relation can be of many forms; it can be one-to-one, many-to-one, or many-tomany [8]. Optimally, the relation is many-to-one, where the knowledge about the counterpart is based on a combination of several sources' experiences. However, a distinction of the knowledge credibility according to sources' trustworthiness is required. This paper considers a three-level hierarchy of knowledge sources: a personal opinion, trusted agents' opinions and a public opinion.

\subsection{Personal, Trusted and Public Opinions}

As when considering humans, trust between P2P-networked agents should equally count on the capability of distinguishing between trust derived from different matters and events. Deducing personal opinions based on personal experiences is essential. However, in some situations the observations cannot cover adequate knowledge and relying on others' judgements is necessary. The trusted agents' opinions are "advises" and acquired gathering information by inquiring friendly sources. A public opinion is one reflecting the majority's opinion concerning the matter. Hence, the personal opinion is a concern that is alterable only by the possessing agent. Moreover, each agent should contribute in providing and maintaining a public opinion and collaborate with personally trusted agents to enforce understanding about the counterpart, which 
is/are considered advises and trusted conditionally. Thereby, the agents form sets of reciprocal trusted conglomerates.

The public opinion does not alone solve any of the problems mentioned. It should be considered as we consider, for example, reviews at epinions.com [9]. Therefore, the public opinion can, at most, mend the uncertainty left by the trusted and personal opinion. However, uncertainty should have primary influence on the decision concerning selecting the agent to process the event. This is motivated by the threat of a colluding set of malicious agents collaborating in building a benevolent public opinion.

Because of the anonymity and egocentric behaviour, it is justified that the personal opinion has greater influence on the final outcome than the trusted agents and public opinions [10]. Consequently, a hierarchy of credibility is formed where the personal opinion mends with the trusted and only then with the public opinion. This hierarchy severely hampers the effect of collusion and deflation. However, the different levels of opinions must not result in conflicts though possibly indicating an opposite outcome, and a method of achieving a consensus is needed. This consensus should handle situations such as, for example, when the public opinion suggests negative assessment while the trusted and personal opinion suggest to affirmative with some uncertainty. In addition, a consensus method of the opinions adjusts the personal opinion to the trusted and public opinions and reduces "obstinacy". The consensus of the different opinions results in a situation equal to inter-human interaction, i.e. that a maliciously behaving agent is capable of taking advantage of the conglomerate of reciprocally trusted agents' benevolence only a finite amount of times.

\subsection{Feedback Formation and Distribution}

Trust relying on a public opinion in $\mathrm{P} 2 \mathrm{P}$ networks is motivated because no single entity can have accurate information about all others' conducts. Initially, no data concerning the counterpart exist, suggesting that reputation has to be built from some state. The state of no reputation, and thus the initial state, is considered in this paper as the state of uncertainty; because modelling trust in dynamic networks cannot allow confusion between "don't trust" and "don't know" [11].

A feedback is the generated data concerning the provider of the resource(s) after an event. The generated data is stored locally and submitted to the supervising agent including the ID of the counterpart, a timestamp, the feedback score and the ID of the reporter [12]. Additional application-specific data can be added. Including IDs in the feedback provides a possibility to identify and verify the transaction. In addition, the agents should monitor their reputation and when disagreeing on an evaluation, change the personal opinion about the reporting source accordingly. The timestamp enables utilisation of a degrading formula, with the justification that attitudes can change over time. The feedback itself is graded with a triplet of values; belief $(b)$, disbelief $(d)$ and uncertainty $(u)$. As discussed in section 1.2 and because the metrics are in contradiction and complete equal 1 , their sum must equal 1 .

Considering the definition of a P2P network, the feedbacks must be stored on the connected (live) agents. As a countermeasure for colluding inflation, the agents supervising feedbacks concerning any given agent should perpetually change. Moreover, the agent the feedbacks concern should not be included in the lookup chain 
of locating the supervising agent. Therefore, the feedback supervising agents must be known by all participants all the time. Enabling this in a system utilising Pastry DHT is possible by having the trust supervising agents' IDs dynamically assigned by a hard-coded function in the application. This requires the DHT to assign the IDs dynamically on a per-session basis as a countermeasure for colluding alternation. However, the need of a unique static ID for each participant compels usage of two interconnected DHTs, each consisting of $\mathrm{x}$ tier to maintain scalability. In such a system, one layer provides the static nodeID while the other layer accounts for proximity selection, lookups and the feedback, being dynamically assigned, hereafter denoted sessionID $(s I D)$. This way the needs of a static unique ID and the requirements for countermeasures are satisfied.

Requiring any reporter to file the feedback to, for example, two closest supervising agents of its own $s I D$, would provide data redundancy. That is, if $s I D c<d<e<f$, the agent with $s I D e$ files reputation regarding $s I D c$ to $s I D d$ and $s I D f$. A possible recovery can be conducted by a logical expression, where peer $g, h, i, j$ and $k$ represents adjacent supervisors for $x$, according to the distribution. $i$ 's stored data can be retrieved by data $h \vee j \wedge \neg g \wedge \neg k$. In other words, if $s I D i$ fails, its data can be recovered by summarising all data that $s I D h$ or $s I D j$ store and that are not stored by $s I D g$, nor by $s I D k$.

Moreover, the redundancy provides a way for a newly assigned supervising agent to verify the passed feedbacks. In addition, such $S I D$ data passing provides means for semi-symmetrical distribution. Consequently, in order for colluding inflation to succeed, the malevolent agent should cooperate with the majority of the involved dynamically changing supervising agents. The feedbacks reported to the supervising agents are the values resulting in the public opinion that is a sum, calculated by a subjective logic, for example, the one presented in [13], of all feedbacks from a set of interactions with the agent(s) concerned.

\section{The Incentive}

In reality an incentive is very simple. It is usually money, fame or some other covetous benefit that good performance entitles to. However, distribution of the beneficial is complex. The incentive to be deployed for usage in computerised communication must be based on the idea of giving benefit to the active and benevolent agents and reducing the value of the beneficial as a consequence of unsatisfactory performance. As a result, there must exist a carrot as well as a stick. In order to increase the anticipations truthfulness, experiences should degrade according to time.

\subsection{A Degrading Formula for Trust}

Philosophically, trust can never be absolute [14]. The core idea of this is the fact that even a friend, considered as trustworthy, can fail the expectations; respectively can an untrustworthy agent behave benevolently. To meet these challenges, a degrading formula must weaken the weighs of the feedbacks based on time and sociality. This is necessary in order to give less social agents equal possibilities; weakening recent 
experiences less. Whitby, Jøsang and Indulska [15] proposed a formula without the sociality factor, however, including it in the same formula is easy, resulting in formula 1 .

$$
p_{Z, t_{R}}^{X, t}=\lambda^{t-t_{R}} \gamma^{(l-k)} p_{Z, t_{R}}^{X}
$$

In formula $1, p_{Z, t_{R}}^{X, t}$ is agent $X$ 's rating of agent $\mathrm{Z}$ at time $t_{R}$, $\mathrm{t}$ being the current time. In other words, an event occurred at time $t_{R}$ where agent $\mathrm{X}$ rated agent $\mathrm{Z}$, the current time being t. $0<\lambda<1$ is the longevity factor degrading the rating according to time. The $\gamma^{l-k}$ represents the ordering of the feedback by occurrence, $l$ being the selection's size and $k$ the position number where the most recent is $l$, degrading according to sociality and being $0<\gamma<1$.

The formula should be applied upon the belief and disbelief values in personal opinions' every experience. Because the sum of the metrics is 1 , uncertainty equals $1-b-d$. In addition, formula 1 sociality factor covers the claim that complete trust or distrust cannot exist, and is a countermeasure for key-space depletion, dropping agents with uncertainty exceeding some predefined threshold value. The values assigned for $\lambda$ and $\gamma$ are subject to the application and the environment. The $\gamma$ value should adjust to the frequency of attitude changes; the lower value, the heavier weight on recent events. $\lambda$ depends on the frequency of transactions conducted with the counterpart. $\gamma$ and $\lambda$ combination reacts to changes in attitude and allow the agent to adapt to the environment. Moreover, the degrading formula is forgivable and will grant the maliciously behaving agent a new chance, after a given time, depending on the longevity factor, of acquiring favouring among the reciprocal conglomerate it tried to fool.

\subsection{Calculating with the Metrics}

Calculating and enforcing the accuracy of the metrics is essential in order to reach the decision. Figure 1 illustrates a situation where two trusted agents, Bob and Claire, contribute in enforcing Alice's anticipation concerning the target, David.

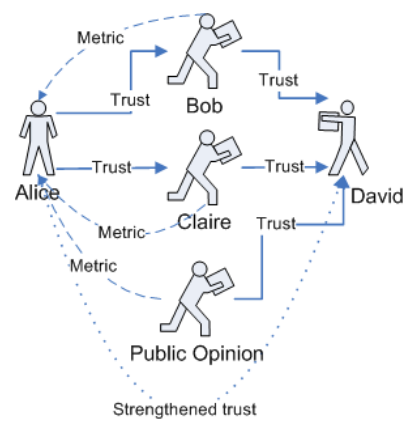

Fig. 1. Trust combination 
The trusted agents participating in the evaluation should contribute with their personal opinions to the requesting entity, without enforcing their understanding by querying further or redirecting. This is motivated because Alice trusts Bob and Claire, not a fourth party, to evaluate David. A situation alike the one in Figure 1 compels a consensus to be achieved between Bob's and Claire's metrics. Bob's and Claire's consensus will eventually be combined with Alice's personal opinion, and finally patched by the public opinion, resulting in the final opinion.

The calculation merging the participating agents' degraded metrics is based on probability calculations and can be performed according to formula 2 illustrated below, originally proposed in [16].

$$
\begin{aligned}
& \text { belief }_{N}^{M}=\left(b^{N} * u^{M}+b^{M} * u^{N}\right) /\left(u^{M}+u^{N}-u^{M} * u^{N}\right) \\
& \text { disbelief }_{N}^{M}=\left(d^{N} * u^{M}+d^{M} * u^{N}\right) /\left(u^{M}+u^{N}-u^{M} * u^{N}\right) \\
& \text { uncertainty }_{N}^{M}=\left(u^{N} * u^{M}\right) /\left(u^{M}+u^{N}-u^{M} * u^{N}\right)
\end{aligned}
$$

$M$ and $N$ are any agents which personal opinion metrics are to be merged; in this case Bob and Claire. If several agents contribute, the merging is done between any two agents or sets of agents at the same level of the consensus process. Eventually the consensuses will reach such magnitudes that it represents the understanding of the underlying group.

The final patching of the uncertainty for the expected outcome utilising the public opinion should be performed after applying the metrics from the trusted agents. This can take place utilising, for example, the following formulas.

$$
\begin{aligned}
& \text { belief }_{\text {public }}^{\text {calced }}=\text { belief }^{\text {calculated }}+\text { uncertainty }^{\text {calculated }} * \text { belief }^{\text {public }} \\
& \text { disbelief }_{\text {public }}^{\text {calced }}=\text { disbelief }^{\text {calculated }}+\text { uncertainty }^{\text {calculated }} * \text { disbelief }^{\text {public }}
\end{aligned}
$$

In these formulas, calculated denotes the degraded trustworthiness of the levels higher in the hierarchy, acquired by formula 2 and 1. Mending this calculated opinion with the public opinion that does not recognise uncertainty, forms an opinion correlating to the expected outcome based on the available knowledge.

Utilising these methods, the trust metric fits the triangle illustrated in Figure 2, when uncertainty is included and the anticipation of forthcoming behaviour is possible. Thus, all possible providers of the requested service can be compared and the most suitable chosen.

\subsection{An Incentive View}

In every incentive method, the inducement must be such that the users cannot gain from reinitiating with a new identity [1]. Hence, we argue that the initial state must be equal to or worse than the state of untrustworthy, with the justification that any knowledge to base anticipation on reducing the risk of misjudgement is better than none. This results in the idea that the state of disbelief is preferred to the initial state, countermeasuring whitewashing.

This paper considers the initial state as the state of uncertainty, a state where no anticipation about future behaviour based on reputation is possible. At the same time, 
the state of uncertainty indicates that the ID is available for any requesting newcomer. The incentive triangle, derived from the opinion triangle in [16], illustrated in Figure 2 , summarises these ideas.

The triangle should be interpreted so that each vertex represents completeness. Therefore, the trustworthiness of any agent consisting of three metrics is representable by one point in the triangle. The median starting at each vertex is the grading of the different values, where belief is represented by $\mathrm{Q}$, disbelief by $\mathrm{R}$ and uncertainty by $\mathrm{P}$. The dot represents an example (personal opinion), with belief (Q) 0.25 , disbelief $(\mathrm{R})$ 0.65 , uncertainty $(\mathrm{P}) 0.1 . \mathrm{E}(\mathrm{x})$ presents the mending (expectation), illustrated in formula 3 and 4, where the personal opinion's uncertainty is mended by the public opinion, whose value is represented by the dotted line $a_{x}$. In Figure 2, this starts at uncertainty, ending at belief $=0.6$ and thus disbelief $=0.4$.

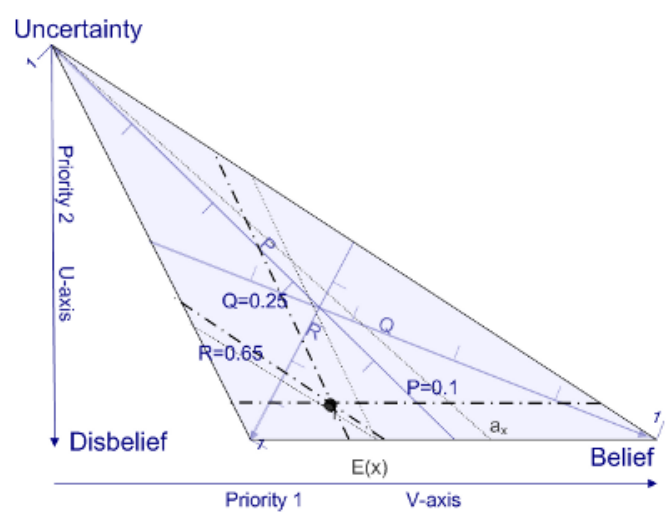

Fig. 2. Incentive triangle

When calculating the expectations value, the final value is required to be either affirmative or negative and thus uncertainty must equal 0 . Uncertainty is reduced to equal 0 by applying the public opinion on formula 3 and 4, resulting in the removal of the uncertainty metric. The degrading formula 1 affects the opinion in the way that it moves towards uncertainty on the axis with the original relation between trust and distrust. Moreover, the triangle recognises two priorities, which are determined by trust qualities and thus purpose specific.

In this specific view, a newcomer is not assigned any profit, which should be the best countermeasure for avoiding an agent with bad reputation to reinitiate its trust relation in form of signing in with a different ID. This implies that the participants are encouraged to consistently act using the same identity every time.

The presented ideas maintain a balance between capability to operate and actual trustworthiness. If some agent is incapable of fulfilling the placed expectations, its trustworthiness will suffer among the expecting agents. Consequently, the network has reacted to this successfully and the trustworthiness/capability balance is maintained. 


\section{Conclusion}

Combining the models presented in this paper reduces the presented problems' severity. Colluding inflation can occur a finite amount of times per conglomerate of reciprocally trustworthy agents because of the influence of the personal opinions. Deflation compromises the public opinion but the target maintains its ability to operate due to the personal opinions and will recover because of the degrading formula. Issuing countermeasures for faking is very difficult, if not impossible, without pre-shared secrets or intermediate authenticating servers. The "free-rider" and the "tragedy of the commons" problems are solved by a carrot - stick relation and utilisation of the personal and public opinion. In addition, the presented incentive reacts to changes in attitude and provides a possibility for malevolent/passive behaviour to change without re-identification.

The problems remaining are the evidence concerning a feedback and the assigning of a unique ID. These issues are of different character and we cannot see the way these could be solved utilising an incentive. Moreover, credentials are excluded from this paper, but being an extension of trust relationships, they are an essential part of trust in reality.

Any accurate simulations to enforce the claims in this paper are difficult to make because the contribution is in anticipation of the irrational. Simulations can thereby not reach greater accuracy than having a static value to calculate irrationality from, which is superficial. The reason is that this would imply simulating human behaviour, but since the human society is functional, creating a similar environment for computerised communication should be the objective. This paper has provided some ideas in order to reach this objective from the point of view that nature has evolved the ultimate trust formation scheme.

\section{References}

1. Spandar D. Kamvar, Mario T. Schlosser, Hector Garcia-Molina (2003). 'The EigenTrust algorithm for reputation management in P2P networks". In Proceedings of the Twelfth International World Wide Web Conference, May, 2003.

2. Rydiger Schollmeier (2002). "A Definition of Peer-to-Peer Networking for the Classification of Peer-to-Peer Architectures and Applications". Proceedings of the First International Conference on Peer-to-Peer Computing (P2P'01).

3. Dan S. Wallach (2002). "A Survey of Peer-to-Peer Security Issues". International Symposium on Software Security (Tokyo, Japan), November 2002.

4. Miguel Castro, Peter Druschel, Y. Charlie Hu, Anthony Rowstron (2002). "Exploiting network proximity in peer-to-peer overlay networks". Technical Report MSR-TR-2002-82 (2002).

5. Kevin Walsh, Emin Gun Sirer (2005). "Thwarting P2P Pollution Using Object Reputation". Cornell University, Computer Science Department Technical Report TR2005-1980.

6. YangBin Tang, HuaiMin Wang, Wen Dou (2004) "Trust Based Incentive in P2P Network". Proceedings of the IEEE International Conference on E-Commerce Technology for Dynamic E-Business (CEC-East'04). 
7. eBay.com. "Understanding feedback scores". URL: http://pages.ebay.com/help/feedback/ feedback-scores.html

8. Tyrone Grandison, Morris Sloman (2000). "A survey of trust in internet applications". 4th Quarter 2000 issue of IEEE Communications Surveys \& Tutorials.

9. Epinions.com. http://www.epinions.com

10. Vinny Cahill, Elizabeth Gray, Jean-Marc Seigneur, Christian D. Jensen, Yong Chen, Brian Shand, Nathan Dimmock, Andy Twigg, Jean Bacon, Colin English, Waleed Wagealla, Sotirios Terzis, Paddy Nixon, Giovanna di Marzo Serugendo, Ciarán Bryce, Marco Carbone, Karl Krukow, Mogens Nielsen (2003). "Using trust for secure collaboration in uncertain environments". IEEE pervasive computing, volume 2, number 3, July September 2003, page $52-61$.

11. Marco Carbone, Mogens Nielsen, Vladimiro Sassone (2003). "A formal model for trust in dynamic networks". BRICS Report RS-03-4, 2003.

12. Li Xiong, Ling Liu. "PeerTrust: Supporting reputation-based trust for peer-to-peer electronic communities". IEEE Transactions on knowledge and data engineering, vol. 16, no. 7, July 2004.

13. Audun Jøsang (2001). “A Logic for Uncertain Probabilities”. International Journal of uncertainty, Fuzziness and Knowledge-Based Systems. 9(3), pp.279-311, June 2001.

14. Martin Hollis (1998). "Trust within reason". Cambridge, United Kingdom, Cambridge university press.

15. Andrew Whitby, Audun Jøsang, Jadwiga Indulska (2004). "Filtering Out Unfair Ratings in Bayesian Reputation Systems". In the Proceedings of the Workshop on Trust in Agent Societies, at the Third International Joint Conference on Autonomous Agents $1 \&$ Multi Agent Systems (AAMAS2004), New York, July 2004.

16. Audun Jøsang (1997). “Artificial Reasoning with Subjective Logic”. In Proceedings of the Second Australian Workshop on Commonsense Reasoning, 1997. 